\title{
Emergence of High Gloss Polymer Compounds to Replace Paintable Compound
}

\author{
TANG YUHANG*, CHEN GUOXIONG, QIAN ZHIJUN, GUO TANGHUA
}

National-certified Enterprise Technology Centre, Kingfa Science \& Technology. Co., Ltd., National Engineer Laboratory for Plastic Modification \& Processing, GuangZhou, 510663, China

Abstract: This paper describes the mechanism and trend of High gloss polymer compounds replacing of paintable plastic parts in automobile industry, and gives some solution overcoming the appearance defects that Painting free parts form easily flow marks and Weld lines in injection molding.

Keyword: High gloss compounds, paintable compound, flow mark, weld line, countermeasures

\section{Introduction}

With the development of automotive industries and the steadily awareness of the public on the environmental protection, Green Manufacture becomes the trend in the automotive industries [1,2]. As for plastics, it has the advantage in low density, easy to process, anti-erosion, good performance in Noise, vibration and Harshness (NVH) [3], which enrich the design freedom in the part, and as a result, plastics have more and more applications even to some extent, it becomes he first choice in auto industries for interiors as well as exteriors, provided that it can meet the mechanical properties requirement. At the same time, it is also true that to avoid degradation in plastic, Painting has to be employed to achieve the good weathering resistance and better aesthetics as in the extreme application such as bumper or Instrument panel, which plays an important role in the scratch resistance as well. In the painting process, right volatile solvents have to be adopted for dilution or curing, which create huge pressure on the environment and operators' healthy/occupational safety. Due to the incompatibility of painting (Thermal set plastics in most case) and plastics, it creates another issue in the salvage of NG parts, and the cost increase in this process subsequently. So minimizing the cost of painting process is important. Recently, technical innovation in painting free compound has brought revolution in automobile industry.

\section{Materials and methods}

\subsection{Material requirement, mechanism and drawback on high gloss polymer compounds}

\subsubsection{Requirement and mechanism on material}

Painting-free-technology or mold-in-color technology was referred to the comprehensive technologies includes proto part design, material development, mold design and cutting, injection process, etc. The process of painting-free technology is using the right compounding materials to inject at the mold to get the desired part with adjusting to injection molding parameter [4]. Since the finished part was molded in one shot and no painting is required, it has the advantage in terms of lower I/O ration, being pollution-free, having short cycle time in injection molding and the most important of all, the easy to recycling. etc. Compared with painting process, one of the latest examples of application advanced compounds on component of automobiles, the cost has reduced almost 30 to $45 \%$.

In fact, painting free materials was compounded by selection some special pigment or additives. Material is tailor-made by fine-tuning the formulation and optimizing compounding parameter in twin screw extruders. The selected additives and pigments are mainly of laminar structure colorants have bigger length/ thickness ratio (as Figure 1 SEM photo of metallic pigment). These colorants are produced by surface treatment, to enhance the adhesion between matrix and also promote color effect of final product. By such treatment, the properties deterioration was relieved. From the existing applications, most preferred colors in the automotive industry are metallic shades, although the requirement of

\footnotetext{
*email: tangyuhang@Kingfa.com.cn
} 
final effect in different for each OEM and varies a lot.

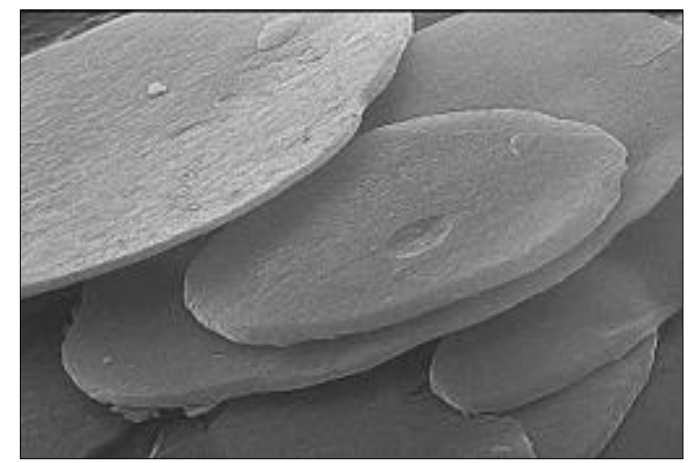

Figure 1. SEM photo of metallic pigment in plastics

From the BOM design point of view, it can be found that materials with better flow will have better metallic, pearl and shining effect like PP with Talc, heat resistant ABS, PC/ABS, Mineral filled PA. On the other side, the material reinforced by glass fiber will have poor performance due to the orientation of glass fiber which covers the pigment in the material as well as the higher content loading of glass fiber in the compound.

\subsubsection{Advantage and defect of painting free materials}

From the introduction given above, it can be seen that for painting free materials, ensuring consistent orientation of colorants/filler/additives is the key for final esthetics effect. Figure 2 Illustration of light refection in plastics. Here it can be seen the more of dispersion of pigment in flat orientation, the better metallic effect it will be provided each other keep the same.

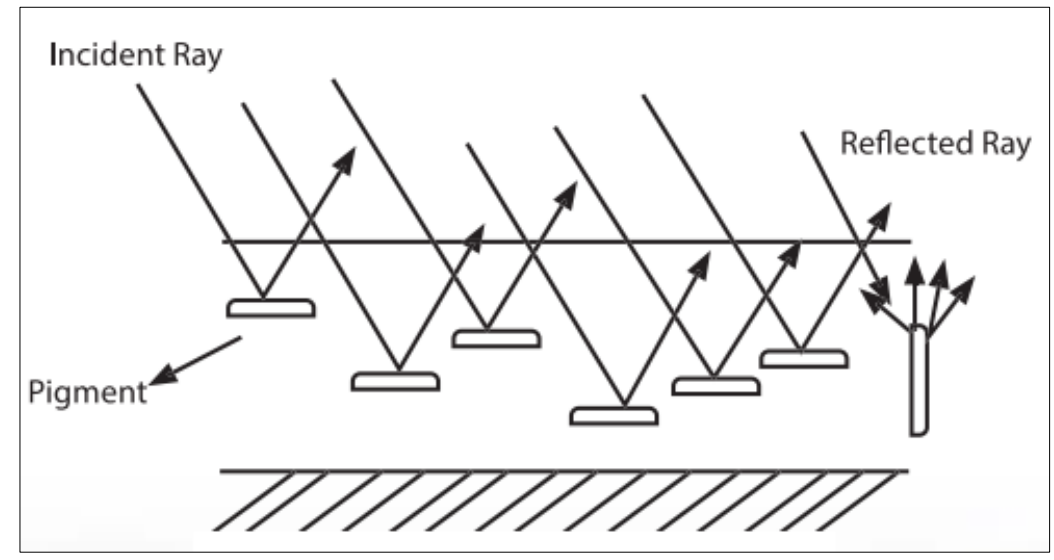

Figure 2. Illustration of light reflection in plastics

The final product is obtained by injection molding at one time, it has many advantages, such as high yield, no pollution, short molding cycle, easy recovery and so on. However, compared with the painting process, its main disadvantages are as follows:

1) In the actual application, due to the part design and limitation of injection parameters, meeting of two different flow is not avoidable in the part so that when the meeting line is within the range of some angle, welding line was formed. In the painting free material, the orientation of pigment will increase the visibility of welding line sometimes it even becomes unacceptable for customer;

2) The injection molding process window is narrow, which requires high stability of the material. The slight deviation between the dispersion of colorant and the flow of material will affect the appearance. 


\section{Results and discussions}

\subsection{Countermeasures for flow mark / weld lines}

In the injection molding process of plastic parts, flow mark is a common defect due to the defects of parts design and injection molding process [4]. Secondly, it is easy to form obvious convergence line while two molten meets with certain angle, which is the weld line defect as well [5-7]. In high gloss polymer compounds, the different orientation of metal Colorant will enlarge the aesthetic defects of weld line appearance, even it is not be accepted totally to consumers. Therefore, it is very critical to how to avoid the formation of flow marks and weld lines or transfer them to invisible and unimportant positions through process, design and materials. Here are some feasible solutions for your reference.

\subsection{Design principle of prototype}

Reasonable selection of prototype. Generally speaking, the application scope of high gloss compounds is not unlimited. Prototype need to be evaluated before high gloss polymer compounds adopted. The common principles are as follows:

1) Try to choose the prototype without holes, because the holes will inevitably lead to the occurrence of weld lines.

2) Try to select the prototype without massive of Snap Joint, reinforced rib and guide pillars on the back. Figure 4 shows the appearance defects such as flow marks and shrinkage marks caused by more snap joint and ribs on the back of the parts.
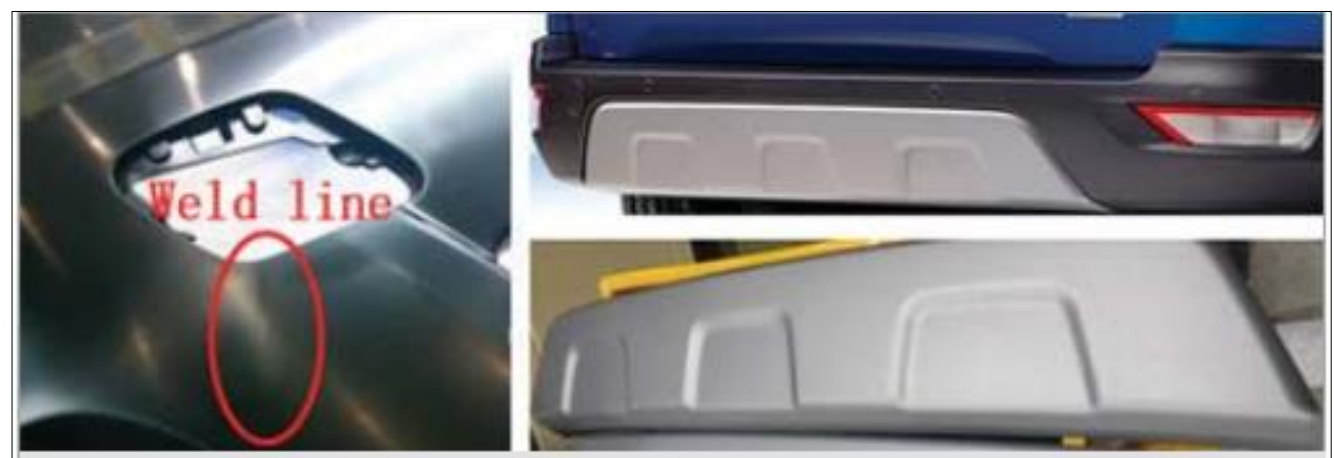

Figure 3. Weld lines effect on the surface

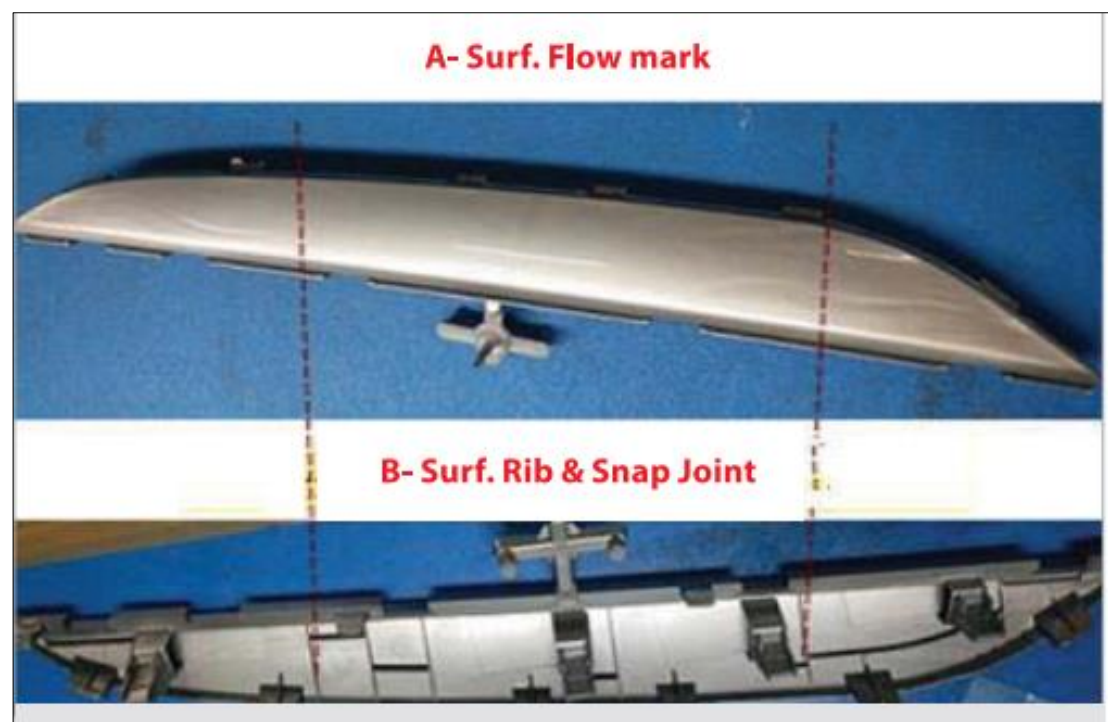

Figure 4. Flow mark and sink mark on the rib structured part

3) Try to selection the part with grains, Figure 5 shows that a good grains surface design can cove up the appearance defects. 


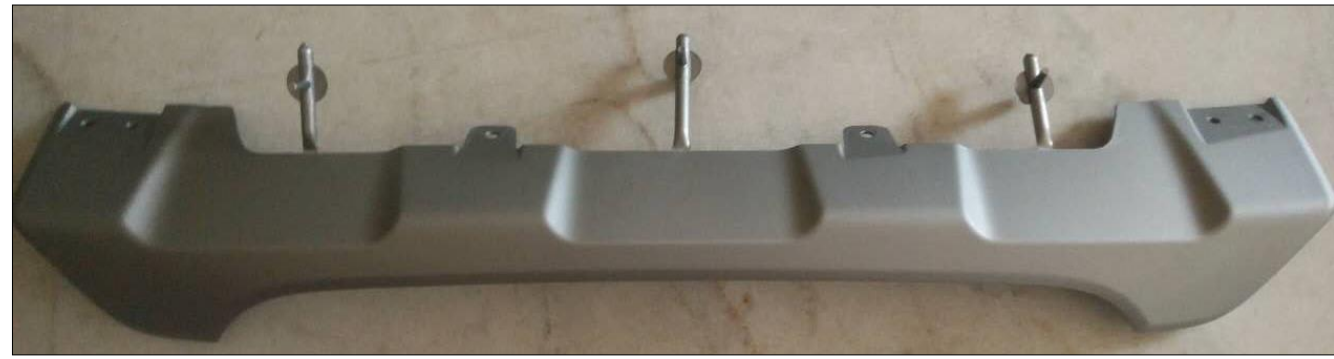

Figure 5. Grains part with good texture

4)Part with even thickness or no drastically change in the wall thickness, and the gradient of melt mass flow rate should be as consistent as possible.

5)Requirement of heat resistance and weathering properties is relatively high.

Figure 6 shows that compared with the common materials of the same grade, the high gloss polymer compounds material performs better in terms of gas generation and color change

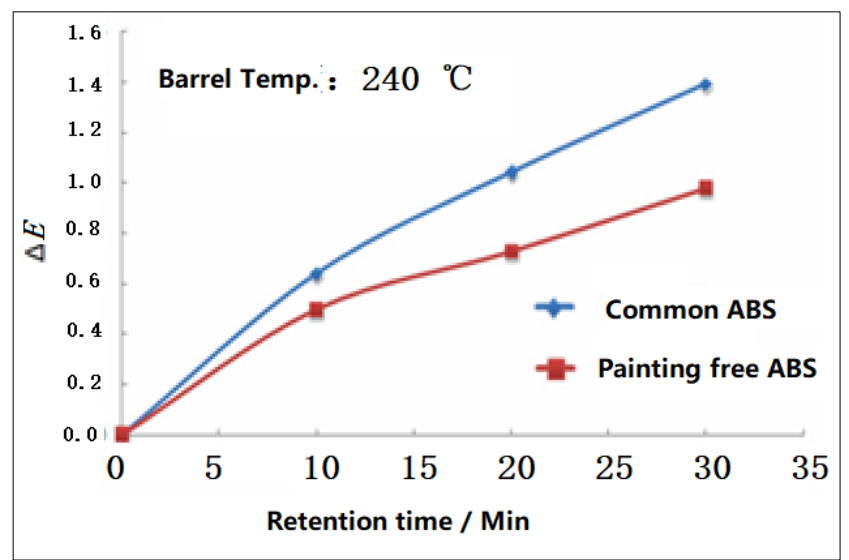

Figure 6. Relationship between color vary and retention time in Barrel

6) Scratch resistance. Table 1 shows pencil hardness of High gloss ABS compounds, common ABS and spraying ABS material. The results show that the scratch resistance of high gloss ABS compounds is better than that of common ABS material, but weaker than that of spraying ABS material.

Sheet 1. Pencil hardness of different material

\begin{tabular}{|c|c|c|c|c|c|}
\hline & Pencil Hardness & $\begin{array}{c}\text { Ball scratch profile } \\
\text { BSP with (um) }\end{array}$ & & $\begin{array}{c}\text { Pencil } \\
\text { Hardness }\end{array}$ & $\begin{array}{c}\text { Ball scratch profile } \\
\text { BSP with (um) }\end{array}$ \\
\hline $\begin{array}{c}\text { High gloss Polymer } \\
\text { compounds }\end{array}$ & F & 300 & $\begin{array}{c}\text { Common } \\
\text { ABS }\end{array}$ & $2 \mathrm{H}$ & 280 \\
\hline Common ABS & B & 320 & & \\
\hline
\end{tabular}

From the above principles, the application of High gloss polymer compounds technology is mainly concentrated in the fields of grille, side wall, bottom guard plate, part of decorative interior, luggage rack, license plate and so on.

\subsection{Reasonable treatment in the welding line}

Although welding line is not desired for painting free but it is not possible to eliminate in all the application even by selection the part. So how to solve this conflict is important and key factors to make success in application. 
1) Flat fan gate and straight gate should be used as far as possible to reduce the impact on material flow, and improve the stability of material flow for fully showing the color effect. One gate should be considered replacing of two gates. While multi-gates are not inevitable, Gate Design of the sequence valve should be adopted, meantime, the injection molding process to be adjusted adaptively accordingly, especially the opening time of the subsequent valve is adjusted slightly. Figure 7 shows the weld line defect caused by improper opening time of sequence valve.

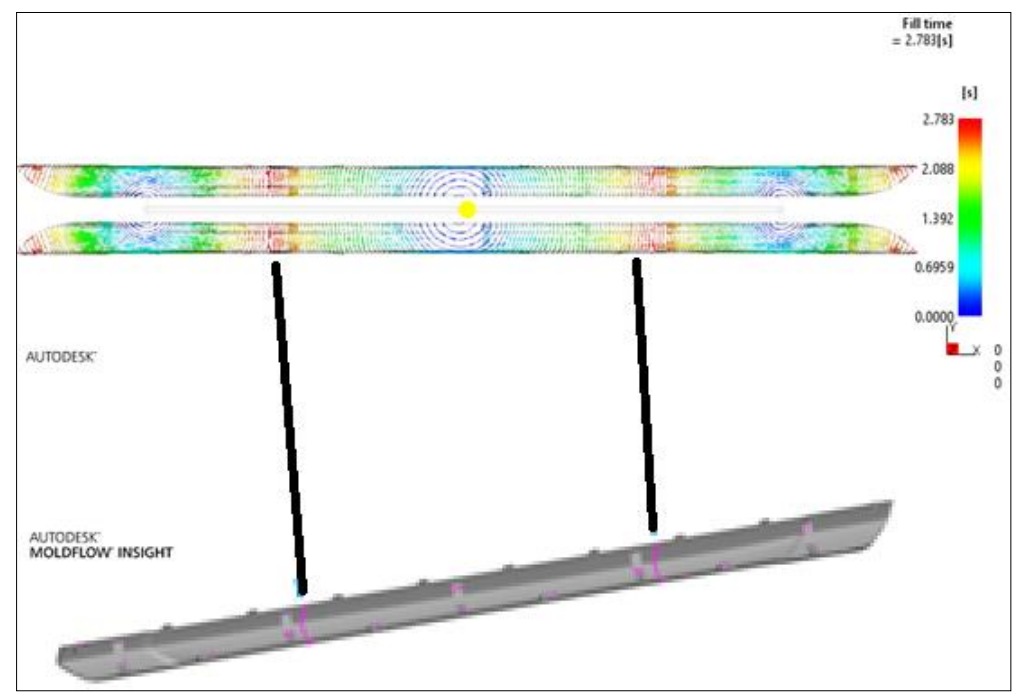

Figure 7. Ill-suited opening time of sequence valve

2) Shift the weld lines to an invisible position.

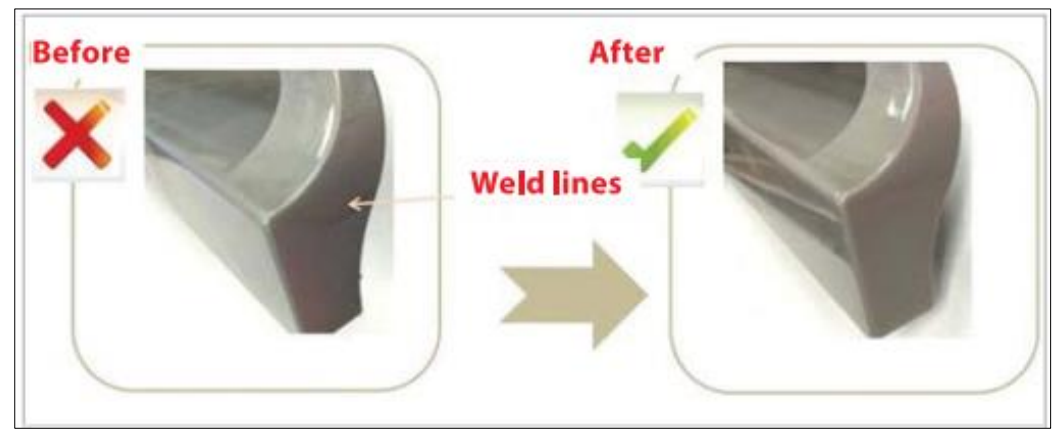

Figure 8. Shift the welding line to invisible area

a) Transfer the weld lines to non-decorative surface, such as gate location, illustrations showed as Figure 8.

b) Add some necessary post process such as thermal printing, imprinting, Water transfer printing, etc. Such process is also must for the final part. One example is in the decoration part in the IP, except silver metallic effect part, water transfer printing is also deployed to add more features on the part, or stick the logo to the welding area to cover.

c) Integration with assemble process. Consider the assemble, by changing the mold design the welding line was shift to the fixture area, which will be covered by post part in the assemble.

d) Add slug well in the final filling area. The welding line will be pushed to this slug well area which can be removed after injection so that in the part no welding line was there, Illustrations showed as Figure 9. 


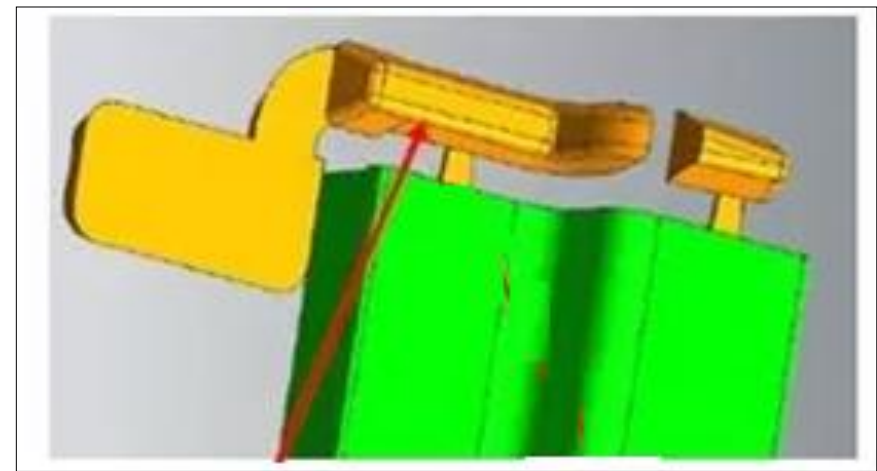

Figure 9. Cold Slug well at the extremes of the part

e) With adoption of special gate such as cutting-in-mold or central removable gate to remove the welding line. In the first case, after the mold was filled, the gate area was punched to reduce the thickness of gate area before the cooling of melt flow. After the part was taken out, some labor was provided to remove the punching area. As the result of punching, the thickness of wall was reduced, and it can be removed by some tools or even with figures. So almost no effect on the productivity. This punching-inmold technology can provide a good solution for the welding line in the gate area, and shows its superiority in the industry compared with other methods. Illustrations showed as Figure 10.

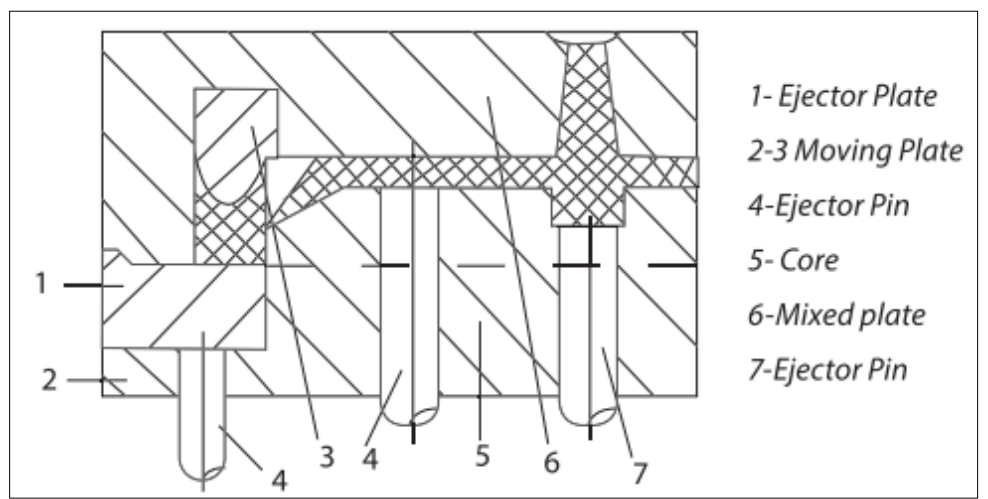

Figure 10. Special gate such as cutting-in-mold

Reasonable grains or texture on the mold. Due to the impact of texture on the flow, texture can change the flow details effectively, and reduce the welding line visibility. In the actual conditions, selection of right grains also play an important role in the aesthetics. Random texture or grains with reasonable depth will lead to better effect than the texture with high gloss. In this case, texture should be considered from the design stage. Below is one example for the effect of same materials on the different grains. It can be seen that even simple grains reduce the visibility of welding line compared with high gloss mold. Illustrations showed different depth and draft angle of grains samples as Figure 11. 


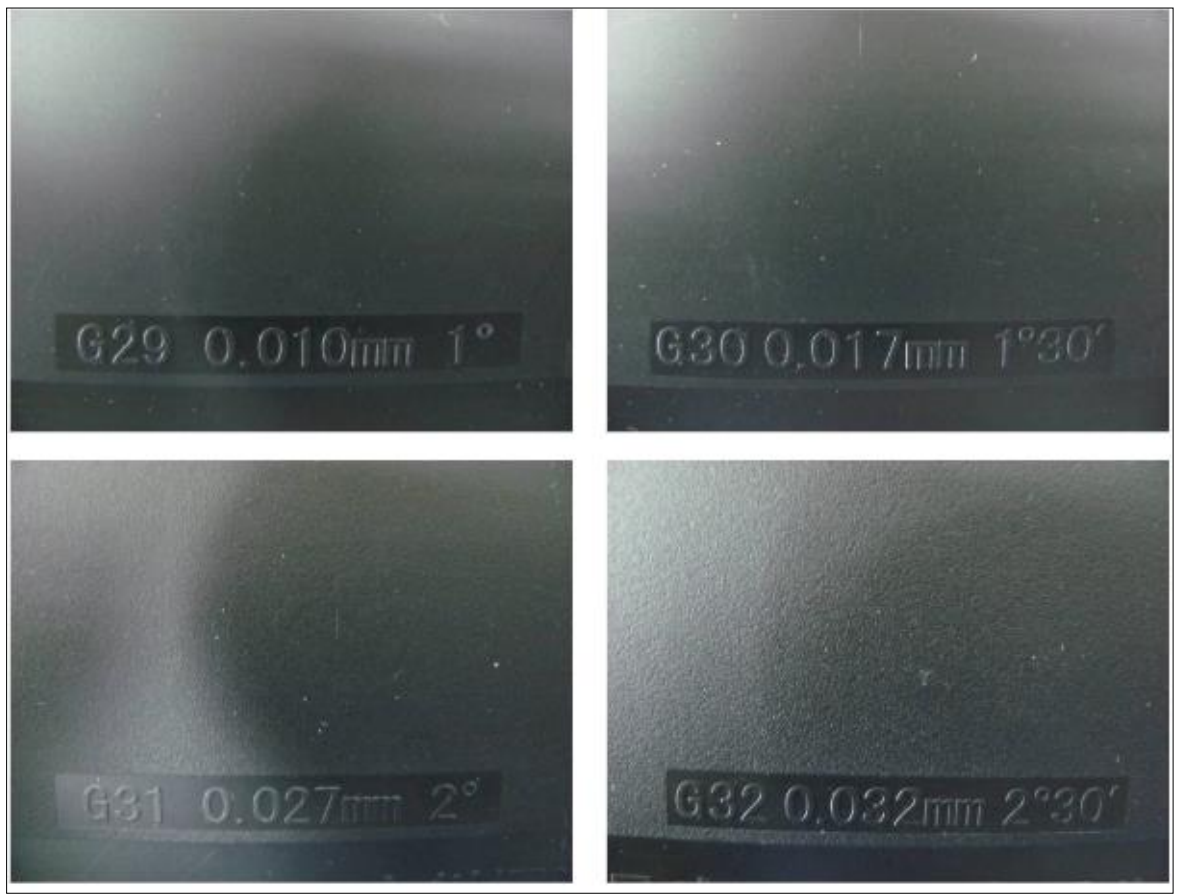

Figure 11. Sample Grain with Diff. Depth and Draft

\subsection{Countermeasures for material and process technology}

1) Selection of Raw material. Making some fine-tune in the compounding to increase the flow and decrease the length of welding line is an important method. At the same time, modification on the gloss /tiger mark performance /selection of filler, may lead to better result of visibility as well. The principle of material formulation design is increasing the flow stability of material in the mold and reducing the turbulence.

2) Right injection parameter by cooperation of injection speed as well as molding temperature. Generally speaking, higher injection speed has better performance in the welding line by keeping the melt temperature but in this case, due to the turbulence caused by high flow, the orientation of pigment will be not so good for aesthetics surface, and also increase the possibility for tiger mark/venting/gas mart. So multi-stage injection speed method has to be employed along with the opening setting of sequential gate/ High temperature fast cooling mold. Meanwhile, mold temperature should be considered also. Higher temperature can be adopted provided that material has good heat stability. Illustration as Figure 12 displays the appearance of injection molding products under different process parameters.
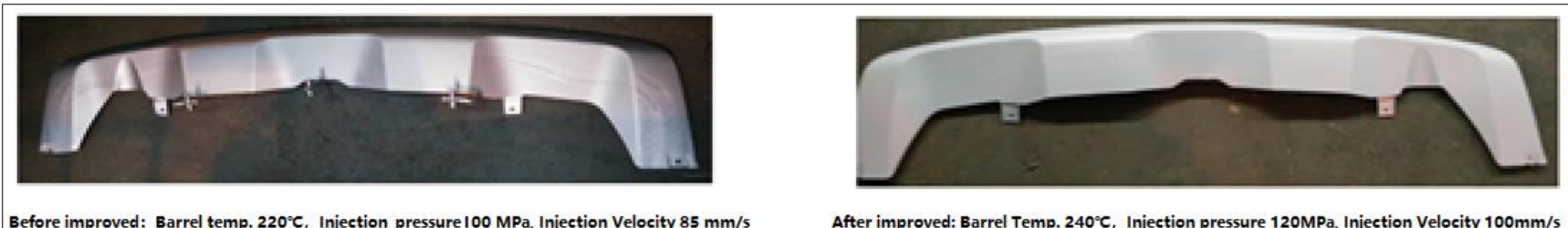

After improved: Barrel Temp. $240^{\circ} \mathrm{C}$, Injection pressure $120 \mathrm{MPa}$, Injection Velocity $100 \mathrm{~mm} / \mathrm{s}$

Figure 12. Influence of process optimization on the appearance of components

High gloss compounds technology as a new fast developing green production method, it can reduce the cost effectively and improve the competitiveness of products. But it is not panacea for all the application, make a reasonable choice according to the above points in order to obtain greater economic benefits. 


\section{Conclusions}

In order to protect the environment, low-cost system and green manufacturing are the future of the development of automobile industry. With the in-depth research and development of high gloss compounds technology, it is more and more popular and commonly to replace of painting parts. However, compared with painting parts, some defect of high gloss compounds technology still needs to be overcome and developed: 1) the scratch resistance of the surface is related to the selected materials, from hardness of $\mathrm{h}$ to $2 \mathrm{~B}$, none of them can reach the hardness of painting parts. 2) it is difficult to achieve special effects such as multi- color coexistence and gradual variation. 3) The color effect that can be achieved is limited, mainly concentrated in silver, pearlescent, etc. 4) There are few theoretical studies on injection molding of high gloss polymer material from compounding technology, lack of corresponding material constitutive equation to simulate the fluidity and appearance effect, and only MoldFlow software for relatively simple analysis.

\section{Reference}

1.MARCELLO C. New trends and developments in automotive industry [M]. University of Sheffield, UK Intech,2016: 366-369.

2.ZHONG Y J, GODWIN P. Biodegradable Polymers and green based antimicrobial packaging materials: A mini-review [J], Advanced Industrial and Engineering Polymer Research, 2020 (01):27-35. 3.ZHANG M. Analysis and research on NVH performance of automobile interior [J]. Automobile Applied Technology, 2019 (19: 138-143.

4.GRILLET A M, ARJEN C B, GERRIT W M, FRANK P T. Numerical Analysis of flow mark surface defects in injection molding flow [D]. Netherlans: Eindhoven University of Technology, 2002,46: 651653.

5.YONG H, CHOJAE H, KIM M G, Lee B M. Prevent weld line defects on an automotive crash pad by local heating and cooling with 3D printed insert core[J]. Journal of Mechanical Science and Technology, 2019,33: 2353-2361.

6.CHENG H W, WAN J L. Effects of geometry and injection-molding parameters on weld-line strength. Polymer Engineering and Science, 2005 (07): 1021-1030.

7.JAWORSKI M, MATTHEW J. Numerical and Experimental Evaluation of Weld Line Strength Prediction in Injection Molded Parts [D]. University of Massachusetts Lowell, ProQuest Dissertations Publishing, 2018 : 19-30

Mnauscript received: 24.05 .2021 